\title{
MÉTODOS CLÁSSICOS PARA A AVALIAÇÃO DA INCERTEZA DE MEDIÇÃO EM SISTEMAS MULTIVARIÁVEIS
}

\author{
Márcio A. F. Martins* \\ marcio.engquimica@gmail.com
}

\author{
Ricardo A. Kalid* \\ kalid@ufba.br
}

*Universidade Federal da Bahia, Escola Politécnica, Programa de Pós-Graduação em Engenharia Industrial, Salvador, Brasil

\section{ABSTRACT}

Classical methods for the evaluation of measurement uncertainty in multivariate systems

The aim of the present work is to give a brief review of two classical methods for the evaluation of measurement uncertainty in multivariate systems. The theoretical and practical issues of the method based on the law of propagation of uncertainties (MLPU) and of the method based on the law of propagation of probability density functions using Monte Carlo method (MLPP) are presented. In order to elucidate an application of both the MLPU and MLPP methods an illustrative example is also presented.

KEYWORDS: measurement uncertainty, propagation of uncertainties, propagation of probability density functions, Monte Carlo method, multivariate systems.

\section{RESUMO}

O presente trabalho visa demonstrar uma breve revisão dos dois métodos clássicos para a avaliação da incerteza de medição em sistemas multivariáveis. Os aspectos teóricos e práticos do método baseado na lei de propagação de incertezas (MLPU) e do método baseado na lei de propagação de funções de densidade de probabilidade, implementado via o método de Monte Carlo (MLPP), são apresentados. Além disso, um exemplo ilustrativo é apresentado de modo a elucidar uma aplicação de ambos os métodos.

Artigo submetido em 13/11/2010 (Id.: 1221)

Revisado em 17/03/2011, 26/06/2011, 18/09/2011

Aceito sob recomendação do Editor Associado Prof. Flávio Henrique Vasconcelos
PALAVRAS-CHAVE: incerteza de medição, propagação de incertezas, propagação de funções de densidade de probabilidade, método de Monte Carlo, sistemas multivariáveis.

\section{INTRODUÇÃO}

Os métodos abordados pelo Guia ISO (BIPM et al., 2008a), baseado na lei de propagação de incertezas LPU (Law of Propagation of Uncertainties), e pelo seu Suplemento 1 (BIPM et al., 2008b), baseado na lei de propagação de funções de densidade de probabilidade (PDFs), são aplicados em sistemas de medição que possuem apenas uma grandeza de saída - um mensurando; tais sistemas de medição são classificados como sistemas MISO (Multiple Input Single Output). Recentemente, Martins et al. (2010) apresentam um estudo crítico de referencial teórico e prático sobre esses métodos.

Existem alguns sistemas de medição, contudo, em que mais do que um mensurando depende de um conjunto comum de grandezas de entrada e das demais grandezas de saída (mensurandos); esses sistemas são classificados como MIMO (Multiple Input Multiple Output). Os sistemas MIMO de medição podem surgir em modelos da metrologia óptica, acústica e elétrica; ou modelos de processos químicos e petroquímicos.

Na literatura, grande parte dos trabalhos relacionados aos sistemas MIMO de medição utiliza a LPU, a saber: Bich (1996); Kacker and Jones (2003) apresentam uma fórmula simples para avaliar a incerteza de medição de sistemas MIMO compostos por apenas duas grandezas de saída ; Weise (1987); Bich et al. (1993/94); Lira (2002); D’Antona (2004); Wang and Iyer (2005); Martins (2010) e a norma 
alemã DIN 1319-4 (1999) demonstram uma equação mais geral envolvendo um número qualquer de grandezas de saída (uma forma matricial equivalente à abordagem do Guia ISO), denominado de método da lei de propagação de incertezas multivariável, aqui denotado por método MLPU ( Multivariate Law of Propagation of Uncertainties); outras aplicações do método MLPU envolvendo mensurandos compostos de grandezas complexas (sistemas multivariáveis) são também encontradas na literatura: Ridler and Salter (2002); Willink and Hall (2002); Hall (2003); Hall (2004).

Antagonicamente ao método MLPU, a lei de propagação de PDFs multivariável, implementada via o método de Monte Carlo (MCM), aqui denominado de método MLPP (Multivariate Law of Propagation of Probability Density Functions), é menos abordada para a avaliação da incerteza de medição em sistemas MIMO. Alguns trabalhos que utilizam o método MLPP em diferentes aplicações destacam-se na literatura, são estes: Cordero and Roth (2005); Hall (2006); Lira and Grientschinig (2010); Martins (2010); Possolo (2010); Souza (2010).

Embora muitos trabalhos sobre avaliação da incerteza de medição em sistemas multivariáveis esteja disponível na literatura, a padronização desta tarefa é culminada apenas com a publicação recente do Suplemento 2 do Guia ISO (BIPM et al., 2011). Este Suplemento tem por finalidade fornecer orientações de cunho prático para avaliar a incerteza de sistemas MIMO à luz dos métodos MLPU e MLPP.

O escopo do presente trabalho é revisar aspectos teóricos e práticos dos métodos MLPU e MLPP. Este está organizado na forma como segue. A primeira parte descreve a lei de propagação de incertezas multivariável, assim como um procedimento para estimar os intervalos de abrangência individuais dos mensurandos. A segunda parte focaliza a demonstração da lei de propagação de PDFs multivariável sob os aspectos analíticos e numéricos, esse último baseado no MCM; além disso, um procedimento para estimar os intervalos de abrangência individuais dos mensurandos, baseado no método MLPP, também será apresentado. Por fim, as vantagens e as desvantagens de ambos os métodos são delineadas com base nos resultados provenientes de um sistema não linear.

\section{LEI DE PROPAGAÇÃO DE INCERTEZAS MULTIVARIÁVEL}

O método MLPU consiste em avaliar as estimativas e a matriz de covariância das grandezas de saída (múltiplos mensurandos $Y_{j}$ ), que dependem de várias grandezas de entrada $X_{i}$, por meio de $K$ funções de medição, as quais podem ou não ser funções explícitas. Uma representação do sistema MIMO de medição pode ser expressa da seguinte forma:

$$
\left\{\begin{array}{ccc}
f_{1}\left(Y_{1}, Y_{2}, \ldots, Y_{K} ; X_{1}, X_{2}, \ldots, X_{N}\right) & = & 0 \\
\vdots & = \\
f_{K}\left(Y_{1}, Y_{2}, \ldots, Y_{K} ; X_{1}, X_{2}, \ldots, X_{N}\right) & = & 0
\end{array}\right.
$$

Em um sistema de medição representado pela Equação (1), as funções de medição podem ser resolvidas de forma analítica ou numérica; podem ser obtidas através de modelagens fenomenológicas ou empíricas; devem incluir as possíveis compensações sistemáticas provenientes dos sistemas de medição das grandezas de entrada; o número de funções de medição $\left(f_{K}\right)$ deve ser igual ao número de grandezas de saída ou mensurandos. Além disso, tanto as grandezas de entrada quanto as grandezas de saída são-lhes atribuídas variáveis aleatórias; para simplificar a notação, todas as grandezas e as variáveis aleatórias lhes atribuídas são denotadas pelo mesmo símbolo.

Conforme exposto inicialmente neste trabalho, o método MLPU é uma generalização do Guia ISO, dessa forma uma aproximação linear das funções de medição deve ser considerada também nesse método.

Em notação matricial, as funções de medição podem ser escritas de uma forma mais compacta, i.e.,

$$
\mathcal{F}(\mathbf{Y} ; \mathbf{X})=\mathbf{0},
$$

em que $\mathbf{Y}=\left(Y_{1}, \ldots, Y_{K}\right)^{\mathrm{T}}$ representa o vetor das grandezas de saída; $\mathbf{X}=\left(X_{1}, \ldots, X_{N}\right)^{\mathrm{T}}$ representa o vetor das grandezas de entrada; enquanto que o símbolo 0 é um vetor coluna com todos seus elementos iguais a zero e $\mathcal{F}(\mathbf{Y} ; \mathbf{X})$ representa o vetor coluna $\left(f_{1}(\mathbf{Y} ; \mathbf{X}), \ldots, f_{K}(\mathbf{Y} ; \mathbf{X})\right)^{\mathrm{T}}$.

De forma análoga ao Guia ISO, a linearização das funções de medição é realizada através de uma expansão da série de Taylor truncada nos termos lineares em torno das estimativas, tanto das grandezas de saída $\mathbf{y}=\left(y_{1}, \ldots, y_{K}\right)^{\mathrm{T}}$, quanto das grandezas de entrada $\mathbf{x}=\left(x_{1}, \ldots, x_{N}\right)^{\mathrm{T}}$. Dessa maneira, a linearização da Equação (2) fornece a expressão seguinte:

$$
\mathcal{F}(\mathbf{Y} ; \mathbf{X}) \approx \mathcal{F}(\mathbf{y} ; \mathbf{x})+\mathbf{S}_{\mathbf{y}}^{\mathbf{T}}(\mathbf{Y}-\mathbf{y})+\mathbf{S}_{\mathbf{x}}^{\mathbf{T}}(\mathbf{X}-\mathbf{x})=\mathbf{0}
$$

As matrizes de sensibilidade das grandezas de saída $\left(\mathbf{S}_{\mathbf{y}}\right)$ e entrada $\left(\mathbf{S}_{\mathbf{x}}\right)$ contêm as derivadas parciais de primeira ordem de cada função de medição em relação as grandezas $\mathbf{Y}$ e $\mathbf{X}$, avaliadas nas estimativas $\mathbf{y}$ e $\mathbf{x}$, respectivamente. Essas matrizes de sensibilidade $\mathbf{S}_{\mathbf{y}}$ e $\mathbf{S}_{\mathbf{x}}$ são as matrizes jacobianas das grandezas de saída $\left(\mathbf{J}_{\mathbf{y}}\right)$ e entrada $\left(\mathbf{J}_{\mathbf{x}}\right)$, respectivamente, as quais são representadas por 


$$
\mathbf{S}_{\mathbf{y}}^{\mathbf{T}}=\mathbf{J}_{\mathbf{y}}^{\mathbf{T}}=\left(\begin{array}{ccc}
\partial f_{1} / \partial y_{1} & \ldots & \partial f_{1} / \partial y_{K} \\
\vdots & \ddots & \vdots \\
\partial f_{K} / \partial y_{1} & \ldots & \partial f_{K} / \partial y_{K}
\end{array}\right)
$$

$\mathrm{e}$

$$
\mathbf{S}_{\mathbf{x}}^{\mathbf{T}}=\mathbf{J}_{\mathbf{x}}^{\mathbf{T}}=\left(\begin{array}{ccc}
\partial f_{1} / \partial x_{1} & \ldots & \partial f_{1} / \partial x_{N} \\
\vdots & \ddots & \vdots \\
\partial f_{K} / \partial x_{1} & \ldots & \partial f_{K} / \partial x_{N}
\end{array}\right)
$$

Como a estimativa $\mathcal{F}(\mathbf{y} ; \mathbf{x})$ é solução da Equação (3), i.e., $\mathcal{F}(\mathbf{y} ; \mathbf{x})=0, \operatorname{logo}$ a Equação (3) pode ser reescrita como

$$
\mathbf{S}_{\mathbf{y}}^{\mathbf{T}}(\mathbf{Y}-\mathbf{y})+\mathbf{S}_{\mathbf{x}}^{\mathbf{T}}(\mathbf{X}-\mathbf{x})=\mathbf{0}
$$

Caso o vetor das grandezas de saída (Y) seja explicitado da Equação (6), por meio da álgebra matricial, obtém-se a expressão

$$
\begin{aligned}
\mathbf{Y} & =\mathbf{S}^{\mathbf{T}}(\mathbf{X}-\mathbf{x})+\mathbf{y}, \\
\mathbf{S}^{\mathbf{T}} & =-\left(\mathbf{S}_{\mathbf{y}}^{\mathbf{T}}\right)^{-\mathbf{1}} \mathbf{S}_{\mathbf{x}}^{\mathbf{T}} .
\end{aligned}
$$

A aplicação do operador variância vetorial ${ }^{1} \mathrm{em}$ ambos os lados da Equação (7) fornece a lei de propagação de incertezas multivariável, ou seja, essa operação matemática fornece a matriz de covariância das grandezas de saída de ordem $K$ $\left(\mathbf{U}_{\mathbf{y}}\right)$, a partir do conhecimento das estimativas $\mathbf{x}$ e $\mathbf{y}$ e da matriz de covariância das grandezas de entrada de ordem $N$ $\left(\mathbf{U}_{\mathbf{x}}\right)$, conforme a expressão

$$
\mathbf{U}_{\mathbf{y}}=\mathbf{S}^{\mathbf{T}} \mathbf{U}_{\mathbf{x}} \mathbf{S}
$$

Como pode ser observado na Equação (8), o método MLPU consiste em propagar as informações disponíveis das grandezas de entrada para as grandezas de saída - vetor das estimativas $\mathbf{x}$ e a matriz de covariância $\mathbf{U}_{\mathbf{x}}-$, por meio de um procedimento linear, conforme é abordado pelo Guia ISO em sistemas MISO de medição.

Os elementos das matrizes de covariância, tanto das grandezas de saída quanto das grandezas de entrada, representam: a variância (ou incerteza padrão quadrática ${ }^{2}$ ) das grandezas (todos os elementos da diagonal principal) e a covariância

\footnotetext{
${ }^{1} \mathrm{O}$ operador variância de uma grandeza (aleatória) vetorial $\mathbf{Z}$, cuja esperança é $\mathbf{z}$ com PDF igual a $g(\mathbf{Z})$, é proveniente da seguinte expressão: $\operatorname{Var}[\mathbf{Z}]=\mathrm{E}\left[(\mathbf{Z}-\mathbf{z})(\mathbf{Z}-\mathbf{z})^{\mathrm{T}}\right] \triangleq \int_{-\infty}^{+\infty}(\mathbf{Z}-\mathbf{z})(\mathbf{Z}-\mathbf{z})^{\mathrm{T}} g(\mathbf{Z}) d \mathbf{Z}$.

${ }^{2}$ Esta definição é proposta por alguns autores do Guia ISO e seus Suplementos (Cox and Harris, 2003), maiores esclarecimentos também podem ser consultados em Bich et al. (2006).
}

existente entre cada grandeza de saída e cada grandeza entrada (todos os elementos que não pertencem à diagonal principal). Essas matrizes são representadas, respectivamente, como

$$
\begin{gathered}
\mathbf{U}_{\mathbf{y}}=\left(\begin{array}{ccc}
u^{2}\left(y_{1}\right) & \ldots & u\left(y_{1}, y_{K}\right) \\
\vdots & \ddots & \vdots \\
u\left(y_{K}, y_{1}\right) & \ldots & u^{2}\left(y_{K}\right)
\end{array}\right) \\
\mathbf{U}_{\mathbf{x}}=\left(\begin{array}{ccc}
u^{2}\left(x_{1}\right) & \ldots & u\left(x_{1}, x_{N}\right) \\
\vdots & \ddots & \vdots \\
u\left(x_{N}, x_{1}\right) & \ldots & u^{2}\left(x_{N}\right)
\end{array}\right) .
\end{gathered}
$$

$\mathrm{e}$

A matriz de covariância associada às estimativas $\mathbf{y}$ dos mensurandos Y, expressa pela Equação (8), é o parâmetro quantitativo usado para avaliar a qualidade da medição proveniente dos sistemas MIMO. Entretanto, algumas aplicações industriais e comerciais requerem o conhecimento da incerteza expandida (intervalo de abrangência) do mensurando, conforme descrito pelo Guia ISO aos sistemas de medição com apenas um mensurando. Nos sistemas MISO de medição, o intervalo de abrangência para o único mensurando $Y$ é estimado com base na fórmula de Welch-Satterthwaite (fórmula W-S) (Hall and Willink, 2001), a qual pressupõe que as grandezas de entrada e de saída, igualmente às suas incertezas padrão, devem possuir distribuições gaussianas; além disso, as grandezas de entrada devem ser estatisticamente independentes, conforme discutido por Martins et al. (2010).

Nos sistemas MIMO de medição, uma região de abrangência $K$-dimensional do espaço (não mais um intervalo de abrangência), referente às estimativas $\mathbf{y}$, é necessária para abranger os valores possíveis dos mensurandos $\mathbf{Y}$. Um procedimento equivalente aos sistemas MISO de medição, ou até mesmo outro, para determinar a região de abrangência dos sistemas MIMO de medição ainda não está bem consolidado na literatura. Apesar disso, o trabalho de Willink and Hall (2002) tem sugerido uma técnica que permite estimar uma região de abrangência aproximada para amostras limitadas $(n \leq 5)$ das grandezas de entrada estatisticamente independentes. Outra alternativa é assumir uma distribuição gaussiana multivariada para os mensurandos, o que implica em uma região de abrangência elipsoidal, para qualquer probabilidade de abrangência escolhida.

Do ponto de vista prático é mais interessante conhecer os intervalos (de abrangência) de cada mensurando individualmente do que a região de abrangência formada por estes. De acordo com os trabalhos de Cox and Harris (2003) e de Bich et al. (2006), os intervalos de abrangência individuais para cada mensurando de um sistema MIMO de medição podem ser estimados à luz do procedimento adotado pelo Guia 
ISO, i.e., para cada elemento da diagonal principal $\left(u\left(y_{j}\right)\right)$ da matriz de covariância $\mathbf{U}_{\mathbf{y}}$ é estimado o fator de abrangência $k_{j}$ com base nos graus de liberdade efetivos de $u\left(y_{j}\right)$, usando a fórmula $\mathrm{W}-\mathrm{S}$, e a probabilidade de abrangência $p$ escolhida. Dessa forma, cada grandeza de saída $Y_{j}$ possui seu intervalo simétrico de abrangência da seguinte forma: $I_{j}=\left[y_{j} \pm k_{j} u\left(y_{j}\right)\right] \equiv\left[y_{j}-k_{j} u\left(y_{j}\right) ; y_{j}+k_{j} u\left(y_{j}\right)\right]$.

\section{LEI DE PROPAGAÇÃO DE FUNÇÕES DE DENSIDADE DE PROBABILIDADE MULTIVARIÁVEL}

O método MLPU utiliza uma aproximação linear das funções de medição para expressar a matriz de covariância associada ao vetor das estimativas das grandezas de saída. Dessa forma, esse método apenas é usado quando as funções de medição são lineares ou linearizadas em torno da região de interesse. Contudo, quando a não linearidade associada ao modelo de medição do sistema MIMO é significativa, um método mais abrangente, que seja aplicado tanto em sistemas lineares quanto não lineares, deve ser requerido de modo a fornecer resultados mais consistentes para a avaliação da incerteza de medição. O método mais abrangente do que o método MLPU é aquele baseado na lei de propagação de PDFs, uma vez que esse fornece uma PDF conjunta para os mensurandos do sistema MIMO de medição. Assim como nos sistemas MISO de medição, a propagação de PDFs nos sistemas MIMO de medição pode ser obtida tanto analítica quanto numericamente.

Nesta seção são demonstrados os métodos analítico e numérico para determinar a PDF conjunta dos mensurandos de um sistema MIMO de medição. Além disso, são delineados os passos para estimar os intervalos de abrangência individuais de cada grandeza de saída desses sistemas.

\subsection{Abordagem analítica}

A essência da lei de propagação de PDFs multivariável é derivar uma PDF conjunta para os mensurandos $\mathbf{Y}\left(g_{\mathbf{Y}}(\boldsymbol{\eta})\right)$, a partir da PDF conjunta das grandezas de entrada $\left(g_{\mathbf{X}}(\boldsymbol{\xi})\right)$ que compõem o modelo MIMO de medição. Neste trabalho, $\boldsymbol{\xi}=\left(\xi_{1}, \ldots, \xi_{N}\right)^{\mathrm{T}}$ denota os valores possíveis das grandezas de entrada $\mathbf{X}$, enquanto que $\boldsymbol{\eta}=\left(\eta_{1}, \ldots, \eta_{K}\right)^{\mathrm{T}}$ denota os valores possíveis das grandezas de saída $\mathbf{Y}$.

Para demonstrar a lei de propagação de PDFs pela abordagem analítica, serão consideradas funções de medição em sua forma explícita, i.e.,

$$
\mathbf{Y}=\mathcal{F}(\mathbf{X})
$$

A esperança do vetor $\mathbf{Y}$ poderia ser obtida através da PDF conjunta das grandezas de entrada $\mathbf{X}$, de acordo com a expressão

$$
\begin{aligned}
\mathrm{E}[\mathbf{Y}] & =\mathrm{E}[\mathcal{F}(\mathbf{X})] \\
& =\int_{-\infty}^{+\infty} \mathcal{F}(\boldsymbol{\xi}) g_{\mathbf{X}}(\boldsymbol{\xi}) d \boldsymbol{\xi}
\end{aligned}
$$

De forma análoga ao procedimento aplicado nos sistemas MISO de medição, ver Martins et al. (2010), a função delta de Dirac é utilizada. Como as propriedades dessa função podem ser aplicadas para grandezas vetoriais, a propriedade de grande utilidade na abordagem proposta será

$$
\int_{-\infty}^{+\infty} \mathbf{z} \delta(\mathbf{z}-\mathbf{a}) d \mathbf{z}=\mathbf{a}
$$

Caso as grandezas vetoriais $\mathbf{z}$ e a sejam substituídas pelas grandezas $\boldsymbol{\eta}$ e $\mathcal{F}(\boldsymbol{\xi})$, respectivamente, então a Equação (13) pode ser reescrita como

$$
\int_{-\infty}^{+\infty} \boldsymbol{\eta} \delta(\boldsymbol{\eta}-\mathcal{F}(\boldsymbol{\xi})) d \boldsymbol{\eta}=\mathcal{F}(\boldsymbol{\xi}),
$$

e a substituição do valor de $\mathcal{F}(\boldsymbol{\xi})$, proveniente da Equação (14), na Equação (12) fornece a expressão

$$
\mathrm{E}[\mathbf{Y}]=\int_{-\infty}^{+\infty} \int_{-\infty}^{+\infty} \boldsymbol{\eta} \delta(\boldsymbol{\eta}-\mathcal{F}(\boldsymbol{\xi})) g_{\mathbf{X}}(\boldsymbol{\xi}) d \boldsymbol{\xi} d \boldsymbol{\eta}
$$

Como a esperança do vetor $\mathbf{Y}$ é definida por

$$
\mathrm{E}[\mathbf{Y}] \triangleq \int_{-\infty}^{+\infty} \boldsymbol{\eta} g_{\mathbf{Y}}(\boldsymbol{\eta}) d \boldsymbol{\eta},
$$

a PDF conjunta das grandezas de saída do sistema MIMO de medição pode ser obtida pela igualdade das Equações (15) e (16), logo

$$
g_{\mathbf{Y}}(\boldsymbol{\eta})=\int_{-\infty}^{+\infty} \delta(\boldsymbol{\eta}-\mathcal{F}(\boldsymbol{\xi})) g_{\mathbf{X}}(\boldsymbol{\xi}) d \boldsymbol{\xi}
$$

Uma vez que a PDF conjunta das grandezas de saída é conhecida, o resultado de medição (estimativa dos mensurandos y) e sua matriz de covariância associada $\left(\mathbf{U}_{\mathbf{y}}\right)$, bem como as PDFs marginais de cada mensurando $Y_{j}\left(g_{Y_{j}}^{\prime}\left(\eta_{j}\right)\right)$, são, respectivamente, 


$$
\begin{gathered}
\mathbf{y}=\mathrm{E}[\mathbf{Y}] \triangleq \int_{-\infty}^{+\infty} \boldsymbol{\eta} g_{\mathbf{Y}}(\boldsymbol{\eta}) d \boldsymbol{\eta} \\
\mathbf{U}_{\mathbf{y}}=\operatorname{Var}[\mathbf{Y}] \triangleq \int_{-\infty}^{+\infty}(\boldsymbol{\eta}-\mathbf{y})(\boldsymbol{\eta}-\mathbf{y})^{\mathrm{T}} g_{\mathbf{Y}}(\boldsymbol{\eta}) d \boldsymbol{\eta}
\end{gathered}
$$

e

$$
g_{Y_{j}}^{\prime}\left(\eta_{j}\right) \triangleq \int_{-\infty}^{+\infty} g_{\mathbf{Y}}(\boldsymbol{\eta}) d \eta_{l} \ldots d \eta_{K}, \quad j \neq l=1, \ldots, K .
$$

Contudo, assim como nos sistemas MISO de medição, a Equação (17) pode ser solucionada analiticamente somente para casos relativamente simples. Portanto, um procedimento numérico, de interesse prático, é necessário para determinar a PDF conjunta dos múltiplos mensurandos que compõem este tipo de sistema de medição. O método numérico mais eficiente para esses sistemas de medição é o MCM, cuja aplicação será demonstrada na próxima seção.

\subsection{Abordagem numérica}

Como demonstrado na seção anterior, integrais multidimensionais (Equação (17)) são exigidas para determinar a PDF conjunta $g_{\mathbf{Y}}(\boldsymbol{\eta})$, cuja solução, geralmente, é realizada por métodos numéricos. O método numérico adotado pelo $\mathrm{Su}$ plemento 1 do Guia ISO é o MCM e, portanto, aqui nos sistemas MIMO de medição uma extensão desse método será adotada.

A ideia básica do MCM, nos sistemas MIMO de medição, é retirar $M$ amostras independentes da PDF conjunta das grandezas de entrada $\left(g_{\mathbf{X}}(\boldsymbol{\xi})\right)$ e propagá-las através das funções de medição (Equação (2)) de modo a gerar $M$ amostras simultâneas das grandezas de saída, conforme a seguinte representação:

$$
\left\{\begin{array}{ccc}
f_{1}\left(\eta_{1}^{l}, \eta_{2}^{l}, \ldots, \eta_{K}^{l} ; \xi_{1}^{l}, \xi_{2}^{l}, \ldots, \xi_{N}^{l}\right) & = & 0 \\
\vdots & = & \vdots \\
f_{K}\left(\eta_{1}^{l}, \eta_{2}^{l}, \ldots, \eta_{K}^{l} ; \xi_{1}^{l}, \xi_{2}^{l}, \ldots, \xi_{N}^{l}\right) & = & 0 \\
l=1, \ldots, M &
\end{array}\right.
$$

Como consequência, uma PDF conjunta empírica para os mensurandos $\left(\hat{g}_{\mathbf{Y}}(\boldsymbol{\eta})\right)$ é construída. Resultados oriundos de $\hat{g}_{\mathbf{Y}}(\boldsymbol{\eta})$ convergem a resultados correspondentes a $g_{\mathbf{Y}}(\boldsymbol{\eta})$ à medida que as amostras de Monte Carlo são grandes (e.g., $\geq 10^{5}$ ).

Como anteriormente enfatizado na seção 2 , os resultados de maior interesse para a avaliação da incerteza de medição de sistemas MIMO são (Bich et al., 2006): o vetor das estimativas do mensurando $\mathbf{y}$; a matriz de covariância $\mathbf{U}_{\mathbf{y}}$; e os intervalos de abrangência individuais $\left(I_{j}\right)$ associados a cada mensurando $Y_{j}$.

As estimativas de cada mensurando $Y_{j}$, bem como as incertezas padrão quadráticas e as covariâncias associadas a essas estimativas, são avaliadas subsequentemente por meio das seguintes Equações:

$$
\begin{gathered}
y_{j}=\frac{1}{M} \sum_{l=1}^{M} \eta_{j}^{l}, \\
u^{2}\left(y_{j}\right)=\frac{1}{M-1} \sum_{l=1}^{M}\left(\eta_{j}^{l}-y_{j}\right)^{2}
\end{gathered}
$$

e

$$
\begin{array}{r}
u\left(y_{j}, y_{m}\right)=\frac{1}{M-1} \sum_{l=1}^{M}\left(\eta_{j}^{l}-y_{j}\right)\left(\eta_{m}^{l}-y_{m}\right), \\
j \neq m=1, \ldots, K .
\end{array}
$$

Os intervalos de abrangência individuais dos mensurandos devem ser obtidos da forma como segue (Bich et al., 2006):

(1) Ordenar em ordem crescente as amostras de cada mensurando $Y_{j}$, i.e., $\left\{\left(\eta_{j}^{(1)} \leq, \ldots, \leq \eta_{j}^{(M)}\right)\right\}$; essas amostras formam uma PDF marginal empírica $\hat{g}_{Y_{j}}^{\prime}\left(\eta_{j}\right)$.

(2) Obter a menor largura possível entre os valores extre$\operatorname{mos} \eta_{j}^{\text {inf e }} \eta_{j}^{\text {sup }}$, para uma dada probabilidade de abrangência $p$. Esses valores extremos são calculados computacionalmente a partir da função de distribuição acumulativa empírica $\hat{G}_{Y_{j}}\left(\eta_{j}\right)$, originada de $\hat{g}_{Y_{j}}^{\prime}\left(\eta_{j}\right)$, que satisfazem a equação $p=\hat{G}_{Y_{j}}\left(\eta_{j}^{\text {sup }}\right)-\hat{G}_{Y_{j}}\left(\eta_{j}^{\text {inf }}\right)$.

Além dos intervalos de abrangência individuais, pode-se também estimar a região de abrangência dos mensurandos a partir da PDF conjunta empírica; um algoritmo para estimar essas regiões está disponível no trabalho de Possolo (2010).

\section{RESULTADOS E DISCUSSÃO}

A partir do anteriormente exposto sobre os métodos MLPU e MLPP, um estudo de caso será abordado nesta seção para elucidar uma aplicação desses métodos de modo a obter maior clareza e assimilação dos mesmos.

No presente estudo de caso, deseja-se estimar a velocidade média e o fator de atrito (fator de fricção) de um fluido, que 
escoa em regime turbulento de um dado trecho reto de tubulação industrial, a partir da queda de pressão (ou perda de carga) existente entre os pontos extremos do trecho reto. Este sistema multivariável (de medição) é tipicamente modelado pelas equações empíricas de Darcy-Weisbach

$$
f_{1}: \Delta P=f \frac{\rho L v^{2}}{2 D}
$$

e de Colebrook-White

$$
f_{2}: \frac{1}{\sqrt{f}}=-2 \log _{10}\left(\frac{2,51}{\operatorname{Re} \sqrt{f}}+\frac{\epsilon}{3,7 D}\right),
$$

em que $\Delta P$ é a queda de pressão no trecho reto da tubulação; $f$ é fator de atrito; $L$ é o comprimento do trecho reto da tubulação; $D$ é o diâmetro da tubulação; $\epsilon$ é a rugosidade da tubulação; $v$ é a velocidade média do fluido; $R e=\rho v D / \mu$ é o número de Reynolds; $\rho$ e $\mu$ são a massa específica e a viscosidade dinâmica do fluido, respectivamente. Essas equações são comumente encontradas na literatura em aplicações da mecânica de fluidos e transferência de calor, e.g., Fox et al. (2009).

As grandezas de entrada $\Delta P, L$ e $D$ são consideradas estatisticamente independentes, ou seja, a matriz de covariância $\mathbf{U}_{\mathbf{x}}$ é diagonal, cujas estimativas e suas respectivas incertezas padrão são apresentadas na Tabela 1. Além disso, as propriedades do fluido e da tubulação (aqui classificados como parâmetros do modelo), $\rho, \mu$ e $\epsilon$, são consideradas possuírem incertezas desprezíveis em relação às grandezas de entrada, cujas estimativas estão disponíveis na Tabela 2.

Tabela 1: Estimativas das grandezas de entrada e suas respectivas incertezas padrão.

\begin{tabular}{lrr}
\hline Grandezas & Estimativa & Incerteza padrão \\
\hline$\Delta P /\left(10^{5} \mathrm{~Pa}\right)$ & 1,5 & 0,1 \\
$L /(\mathrm{m})$ & 50,0 & 0,1 \\
$D /(\mathrm{m})$ & 0,10 & 0,01 \\
\hline
\end{tabular}

Tabela 2: Estimativas dos parâmetros do modelo de medição.

\begin{tabular}{lr}
\hline Parâmetros & Valores \\
\hline$\rho /\left(\mathrm{kg} / \mathrm{m}^{3}\right)$ & $1,0 \times 10^{3}$ \\
$\mu /(\mathrm{Pa} . \mathrm{s})$ & $1,0 \times 10^{-3}$ \\
$\epsilon /(\mathrm{m})$ & $4,5 \times 10^{-5}$ \\
\hline
\end{tabular}

$\mathrm{O}$ conhecimento das informações metrológicas referente às grandezas de entrada, bem como dos parâmetros desse sistema MIMO de medição, permite aplicar os métodos MLPU e MLPP para avaliar a matriz de covariância $\mathbf{U}_{\mathbf{y}}$ e os intervalos de abrangência individuais $\left(I_{j}\right)$ dos mensurandos, conforme procedimento anteriormente exposto sobre os mesmos.

Para aplicar o método MLPP, é necessário estabelecer o número de amostras de Monte Carlo $(M)$ adequado ao modelo de medição. A escolha adequada para $M$ foi obtida mediante a aplicação da análise de sensibilidade das funções de medição, baseada na tolerância numérica associada à matriz de covariância dos mensurandos $\left(\mathbf{U}_{\mathbf{y}}\right)$, maiores detalhes podem ser consultados em Martins (2010). Nesse método foi atribuída uma PDF gaussiana para cada grandeza de entrada $\Delta P, L$ e $D$, cujas esperanças (estimativas) e desvios padrão (incertezas padrão) são aqueles apresentados na Tabela 1 .

Os experimentos numéricos foram implementados em linguagem MATLAB (versão 7.8) sob o sistema operacional Linux (distribuição Ubuntu 10.10), por meio de um PC com 3,0 GHz de frequência, $4 \mathrm{~GB}$ de memória de acesso aleatório $(R A M)$ e um processador Intel Core 2 Duo. O resultado da análise de sensibilidade revelou $M=2 \times 10^{5}$ amostras de Monte Carlo a serem adotadas nesse estudo de caso.

As PDFs marginais dos mensurandos, velocidade média do fluido $(v)$ e fator de atrito $(f)$, geradas pelo método MLPP, são apresentadas na Figura 1.

Os resultados processados pelo método MLPP foram comparados com os resultados provenientes do método MLPU. As estimativas da velocidade média do fluido e do fator de atrito são apresentadas na Tabela 3 , bem como as incertezas padrão, a covariância e os intervalos de abrangência individuais (com base em uma probabilidade de abrangência $p=90 \%$ ) associados a esses mensurandos.

Tabela 3: Comparação entre os resultados oriundos dos métodos MLPU e MLPP.

\begin{tabular}{lrrrr}
\hline & \multicolumn{2}{c}{ MLPU } & \multicolumn{2}{c}{ MLPP } \\
\cline { 2 - 5 } & $v /(\mathrm{m} / \mathrm{s})$ & $f\left(\times 10^{4}\right)$ & $v /(\mathrm{m} / \mathrm{s})$ & $f\left(\times 10^{4}\right)$ \\
\hline$y_{j}$ & 5,91 & 171,84 & 5,90 & 172,17 \\
$u\left(y_{j}\right)$ & 0,42 & 4,33 & 0,43 & 4,45 \\
$\eta_{j}^{\text {inf }}$ & 5,23 & 164,90 & 5,19 & 164,84 \\
$\eta_{j}^{\text {sup }}$ & 6,58 & 178,78 & 6,58 & 179,20 \\
$u(v, f)$ & $-1,66 \times 10^{-4}(\mathrm{~m} / \mathrm{s})$ & \multicolumn{2}{c}{$-1,70 \times 10^{-4}(\mathrm{~m} / \mathrm{s})$} \\
\hline
\end{tabular}

Como pode ser observado na Tabela 3, a diferença entre as estimativas dos mensurandos, bem como os parâmetros metrológicos que caracterizam o grau de confiança dessas estimativas - incertezas padrão, covariância e intervalos de abrangência -, obtidos por ambos os métodos, é praticamente inexistente; a diferença percentual entre os resultados dos métodos MLPU e MLPP é aproximadamente menor do que 

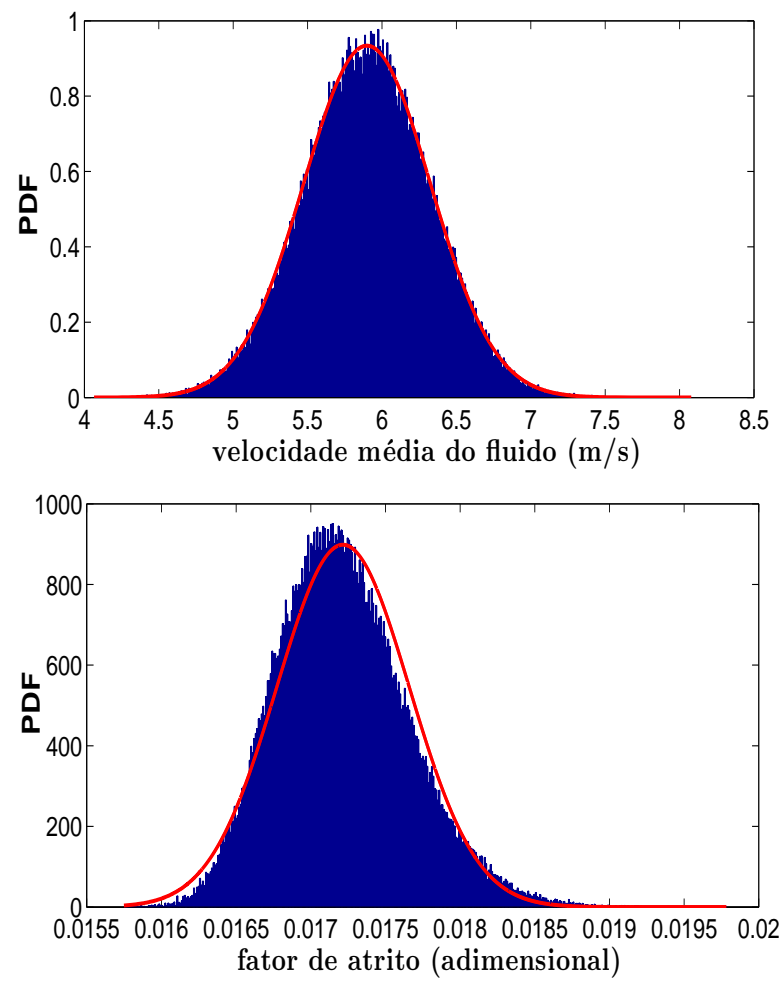

Figura 1: PDFs marginais da velocidade média do fluido e do fator de atrito. A apresentação da curva gaussiana (linha cheia vermelha) indica quanto cada PDF marginal desvia-se de uma PDF gaussiana correspondente, cuja esperança e desvio padrão são iguais à estimativa e à incerteza padrão provenientes do método MLPP.

0,2\%. Dessa forma, quaisquer métodos (MLPU e MLPP) podem ser utilizados para avaliar a incerteza desse sistema de medição.

Apesar dos resultados gerados por ambos os métodos serem praticamente iguais, nesse estudo de caso, o método MLPP é mais consistente do que o método MLPU para avaliar a incerteza de medição, pois esse considera quaisquer não linearidades do modelo de medição, isto é, uma maior confiança associada às estimativas dos mensurandos é encontrada via as incertezas geradas pelo método MLPP. Em outras palavras, o fato de considerar todas as não linearidades das funções de medição faz com que os resultados do método MLPP sejam sempre mais adequados e robustos do que aqueles provenientes do método MLPU, tornando-o um método de referência para comparação dos resultados.

A principal limitação do método MLPP reside no fato de exigir um esforço computacional significativo em relação ao método MLPU, contudo a disponibilidade de computadores cada vez mais rápidos e possantes em processamento e uso da memória $R A M$ torna o método MLPP mais exequível.

\section{CONSIDERAÇÕES FINAIS}

Neste trabalho foram apresentados dois métodos para expressar e avaliar a incerteza de medição em sistemas MIMO: o método MLPU, baseado na lei de propagação de incertezas, e o método MLPP, baseado na lei de propagação de PDFs através do MCM.

O método MLPU pode ser usado para caracterizar a medição de sistemas MIMO devido às seguintes vantagens: primeiro, não há necessidade do conhecimento completo da PDF conjunta das grandezas de entrada, são requeridos apenas o vetor esperança e sua matriz de covariância associada; segundo, possui cálculos simples para avaliar a matriz de covariância das grandezas de saída do sistema MIMO que, por sua vez, podem ser facilmente implementados em qualquer linguagem computacional.

Por outro lado, o método MLPU possui limitações relevantes quanto ao seu uso, o que justifica a necessidade de um método mais robusto para expressar e avaliar a incerteza de medição dos sistemas MIMO. As principais limitações desse método são: quando as funções de medição possuem não linearidades significativas, a expansão em série de Taylor truncada nos termos de primeira ordem deve fornecer resultados inconsistentes ou enganosos; na estimativa dos intervalos de abrangência individuais das grandezas de saída é necessário supor uma PDF gaussiana para os mensurandos $Y_{j}$ e suas respectivas incertezas padrão $u\left(y_{j}\right)$, além disso, as grandezas de entrada e suas respectivas incertezas padrão também devem ser mutuamente independentes e possuírem distribuições gaussianas. Ou seja, a aplicação do método MLPU requer muitas hipóteses que, em certas situações práticas, não podem ser satisfeitas.

A avaliação da incerteza de medição por meio do método MLPP é menos restritiva do que o método MLPU, isto é, esse método requer menos hipóteses para sua aplicação em relação ao método MLPU. As vantagens mais relevantes do método MLPP são: fornece uma PDF conjunta e PDFs marginais para as grandezas de saída; não existem limitações no que tange à natureza não linear do modelo MIMO de medição; diferentemente do método MLPU, o qual sempre considera um intervalo de abrangência gaussiano (simétrico), esse método estima os intervalos das próprias PDFs marginais dos mensurandos, seja essa simétrica ou assimétrica, gaussiana ou não.

Entretanto, o método MLPP também possui limitações, a saber: assim como nos sistemas MISO, a atribuição apropriada da PDF para as grandezas de entrada pode ser difícil 
por causa da imprecisão de dados ou falta de conhecimento dos processos físico-químicos que influenciam o processo de medição; o tempo de processamento pode ser muito longo à medida que se aumenta a complexidade e não linearidade dos modelos de medição; por fim, outra dificuldade reside na geração de números aleatórios para uma PDF conjunta não gaussiana, pois a maioria dos pacotes computacionais disponíveis gera números aleatórios somente para PDF conjunta gaussiana; uma alternativa a essa restrição é recorrer às funções cópulas, maiores detalhes podem ser consultados em Possolo (2010).

A partir do anteriormente exposto sobre as vantagens e as desvantagens dos métodos MPLU e MLPP, pode-se concluir que o método MLPU deve ser usado em sistemas de medição lineares ou linearizados, enquanto que o método MLPP é mais abrangente e pode ser aplicado para quaisquer modelos de medição, inclusive os modelos não lineares.

\section{AGRADECIMENTOS}

Os autores gostariam de agradecer os revisores anônimos pelas suas valiosas sugestões para melhoria deste trabalho. Agradecimentos também são devotados à Fundação de Amparo à Pesquisa do Estado da Bahia (FAPESB) pelo apoio financeiro.

\section{REFERÊNCIAS}

Bich, W. (1996). Simple formula for the propagation of variances and covariances, Metrologia 33(2): 181-183.

Bich, W., Cox, M. G. and Harris, P. M. (1993/94). Uncertainty modelling in mass comparisons, Metrologia 30: 495-502.

Bich, W., Cox, M. G. and Harris, P. M. (2006). Evolution of the guide to the expression of uncertainty in measurement, Metrologia 43: S161-S166.

BIPM, IEC, IFCC, ILAC, ISO, IUPAC, IUPAP and OIML (2008a). Evaluation of Measurement Data - Guide to the Expression of Uncertainty in Measurement (GUM 1995 with minor corrections), Joint Committee for Guides in Metrology, JCGM 100.

BIPM, IEC, IFCC, ILAC, ISO, IUPAC, IUPAP and OIML (2008b). Evaluation of Measurement Data-Supplement 1 to the Guide to the Expression of Uncertainty in Measurement - Propagation of distributions using a Monte Carlo method, Joint Committee for Guides in Metrology, JCGM 101.

BIPM, IEC, IFCC, ILAC, ISO, IUPAC, IUPAP and OIML (2011). Evaluation of Measurement Data-Supplement
2 to the Guide to the Expression of Uncertainty in Measurement-Extension to any number of output quantities, Joint Committee for Guides in Metrology, JCGM 102.

Cordero, R. R. and Roth, P. (2005). On two methods to evaluate the uncertainty of derivatives calculated from polynomials fitted to experimental data, Metrologia 42: 3944.

Cox, M. and Harris, P. (2003). The gum and its planned supplemental guides, Accred Qual Assur 8(4): 375-379.

D’Antona, G. (2004). Measurement data processing using random matrices: a generalized formula for the propagation of uncertainty, IEEE Transactions on Instrumentation and Measurement 53: 537-545.

DIN 1319-4:1999-02, Fundamentals of Metrology - Part 4: Evaluation of measurements; uncertainty of measurement (1999).

Fox, R. W., Pritchard, P. J. and McDonald, A. T. (2009). Introduction to Fluid Mechanics, 7th edn, Jonh Wiley.

Hall, B. D. (2003). Calculating measurement uncertainty for complex-valued quantities, Measurement Science and Technology 14(3): 368-375.

Hall, B. D. (2004). On the propagation of uncertainty in complex-valued quantities, Metrologia 41(3): 173-177.

Hall, B. D. (2006). Monte carlo uncertainty calculations with small-sample estimates of complex quantities, Metrologia 43(3): 220-226.

Hall, B. D. and Willink, R. (2001). Does "welchsatterthwaite"make a good uncertainty estimate?, Metrologia 38(1): 9-15.

Kacker, R. and Jones, A. (2003). On use of bayesian statistics to make the guide to the expression of uncertainty in measurement consistent, Metrologia 40(5): 235-248.

Lira, I. (2002). Evaluating the measurement uncertainty: fundamentals and practical guidance, Institute of Physics Publishing.

Lira, I. and Grientschinig, D. (2010). Bayesian assessment of uncertainty in metrology: a tutorial, Metrologia 47: R1-R14.

Martins, M. A. F. (2010). Contribuições para a avaliação da incerteza de medição no regime estacionário, Mestrado em engenharia industrial, Escola Politécnica da Universidade Federal da Bahia. 
Martins, M. A. F., Kalid, R., Nery, G. A., Teixeira, L. A. and Gonçalves, G. A. A. (2010). Comparação entre os métodos linear e não linear para a avaliação da incerteza de medição, Controle \& Automação 21(6): 557-576.

Possolo, A. (2010). Copulas for uncertainty analysis, Metrologia 47(3): 262-271.

Ridler, N. M. and Salter, M. J. (2002). An approach to the treatment of uncertainty in complex $S$-parameter measurements, Metrologia 39: 295-302.

Souza, L. S. (2010). Propagação de incertezas na reconciliação de dados com restrições lineares, Especialização em automação industrial, Escola Politécnica da Universidade Federal da Bahia.

Wang, C. M. and Iyer, H. K. (2005). Propagation of uncertainties in measurements using generalized inference, Metrologia 42(2): 145-153.

Weise, K. (1987). Treatment of uncertainties in precision measurements, IEEE Transactions on Instrumentation and Measurement 36: 642-645.

Willink, R. and Hall, B. D. (2002). A classical method for uncertainty analysis with multidimensional data, $\mathrm{Me}$ trologia 39(4): 361-369. 